\title{
A Note on Cyclic Codes from APN Functions
}

\author{
Chunming Tang · Yanfeng Qi · Maozhi \\ $\mathrm{Xu}$
}

Received: date / Accepted: date

\begin{abstract}
Cyclic codes, as linear block error-correcting codes in coding theory, play a vital role and have wide applications. Ding in 9 constructed a number of classes of cyclic codes from almost perfect nonlinear (APN) functions and planar functions over finite fields and presented ten open problems on cyclic codes from highly nonlinear functions. In this paper, we consider two open problems involving the inverse APN functions $f(x)=x^{q^{m}-2}$ and the Dobbertin APN function $f(x)=x^{2^{4 i}+2^{3 i}+2^{2 i}+2^{i}-1}$. From the calculation of linear spans and the minimal polynomials of two sequences generated by these two classes of APN functions, the dimensions of the corresponding cyclic codes are determined and lower bounds on the minimum weight of these cyclic codes are presented. Actually, we present a framework for the minimal polynomial and linear span of the sequence $s^{\infty}$ defined by $s_{t}=\operatorname{Tr}\left(\left(1+\alpha^{t}\right)^{e}\right)$, where $\alpha$ is a primitive element in $G F(q)$. These techniques can also be applied into other open problems in 9 .
\end{abstract}

Keywords Cyclic codes · sequences · linear span · minimal polynomials · APN functions

Chunming Tang

School of Mathematics and Information, China West Normal University, Sichuan Nanchong, 637002, China

Yanfeng Qi

LMAM, School of Mathematical Sciences, Peking University, Beijing, 100871, and Aisino

Corporation Inc., Beijing, 100195, China

Maozhi Xu

LMAM, School of Mathematical Sciences, Peking University, Beijing, 100871, China 


\section{Introduction}

As a subclass of linear codes, cyclic codes with efficient encoding and decoding algorithms [8, 12, 19], have been widely applied into data storage systems and communication systems. Hence, cyclic codes attract much attention and have been widely studied. Many research results have been made [3,7,14,17].

Throughout this paper, let $p$ be a prime and $q$ be a power of $p$. Let $r=q^{m}$, where $m$ is a positive integer. A linear $[n, k, d] \operatorname{code} \mathcal{C}$ over finite field $G F(q)$ is a $k$ dimension subspace of $G F(q)^{n}$ with minimum distance $d$. The linear code $\mathcal{C}$ is called a cyclic code if $\left(c_{0}, c_{1}, \cdots, c_{n-1}\right) \in \mathcal{C}$ implies that $\left(c_{n-1}, c_{0}, \cdots, c_{n-2}\right) \in$ $\mathcal{C}$. Let $\operatorname{gcd}(n, q)=1$. Then there exists the following correspondece:

$$
\begin{aligned}
\pi: G F(q)^{n} & \longrightarrow G F(q)[x] /\left(x^{n}-1\right) \\
\left(c_{0}, c_{1}, \ldots, c_{n-1}\right) & \longmapsto c_{0}+c_{1} x+\ldots+c_{n-1} x^{n-1} .
\end{aligned}
$$

Then a codeword $\left(c_{0}, c_{1}, \cdots, c_{n-1}\right) \in \mathcal{C}$ can be identified with the polynomial $c_{0}+c_{1} x+\cdots+c_{n-1} x^{n-1} \in G F(p)[x] /\left(x^{n}-1\right)$. Further, $\mathcal{C}$ is a cyclic code if and only if $\pi(\mathcal{C})$ is an ideal of $G F(p)[x] /\left(x^{n}-1\right)$. Since $G F(p)[x] /\left(x^{n}-1\right)$ is a principal ideal ring, there exists a unique monic polynomial $g(x) \mid\left(x^{n}-1\right)$ satisfying $\pi(\mathcal{C})=\langle g(x)\rangle$. We also write $\mathcal{C}=\langle g(x)\rangle$. Then $g(x)$ is called the generator polynomial of $\mathcal{C}$ and $h(x)=\left(x^{n}-1\right) / g(x)$ is called the parity-check polynomial of $\mathcal{C}$.

Sequences over finite field $G F(q)$ can be utilized to construct cyclic codes 9], that is, the minimal polynomial of a periodic sequence can be used as the generator polynomial of a cyclic code. Let $s^{\infty}=\left(s_{t}\right)_{t=0}^{\infty}$ be a sequence over $G F(q)$ with period $q^{m}-1$. Then there exists the smallest positive integer $l$, satisfying

$$
-c_{0} s_{i}=c_{1} s_{i-1}+c_{2} s_{i-2}+\cdots+c_{l} s_{i-l},(i \geq l),
$$

where $c_{0}=1, c_{1}, \cdots, c_{l} \in G F(q)$ and $c_{i}(0 \leq i \leq l)$ are uniquely determined. The unique polynomial

$$
\mathbb{M}_{s}(x)=c_{0}+c_{1} x+\cdots+c_{l} x^{l} \in G F(q)[x]
$$

is called the minimal polynomial of the sequence $s^{\infty}$ and $l$ is the linear span (also called linear complexity) of $s^{\infty}$, which is denoted as $\mathbb{L}_{s}$. Generally, any polynomial as a constant multiple of $\mathbb{M}_{s}(x)$ is also a minimal polynomial of $s^{\infty}$. Further, $\mathbb{M}_{s}(x)$ and $\mathbb{L}_{s}$ are given by [16]

$$
\begin{aligned}
\mathbb{M}_{s}(x) & =\frac{x^{q^{m}-1}-1}{\operatorname{gcd}\left(x^{q^{m}-1}-1, S^{q^{m}-1}(x)\right)}, \\
\mathbb{L}_{s} & =q^{m}-1-\operatorname{deg}\left(\operatorname{gcd}\left(x^{q^{m}-1}-1, S^{q^{m}-1}(x)\right)\right),
\end{aligned}
$$

where

$$
S^{q^{m}-1}(x)=s_{0}+s_{1} x+\cdots+s_{q^{m}-2} x^{q^{m}-2} \in G F(q)[x] .
$$


Hence, the cyclic code with the generator polynomial $\mathbb{M}_{s}(x)$ is

$$
\mathcal{C}_{s}=\left\langle\mathbb{M}_{s}(x)\right\rangle \text {. }
$$

Then, $\mathcal{C}_{s}$ is an $\left[q^{m}-1, q^{m}-1-\mathbb{L}_{s}, d\right]$ cyclic code. Consequently, the determination of $\mathbb{M}_{s}(x)$ and $d$ is essential for studying these cyclic code, which is the main problem in 9 and this paper.

Generally, it is difficult to determine $\mathbb{M}_{s}(x)$ for a sequence $s^{\infty}$. Ding 9 considered a special class of sequences with the form

$$
s_{t}=\operatorname{Tr}\left(\left(1+\alpha^{t}\right)^{e}\right),
$$

where $\alpha$ is a primitive element of $G F(r), \operatorname{Tr}(\cdot)$ is the trace function from $G F(r)$ to $G F(q)$ and $e$ is an exponent making the monomial $f(x)=x^{e}$ an almost perfect nonlinear function or a planar function. In some special cases, Ding determined $\mathbb{M}_{s}(x)$, which induced a number of classes of cyclic codes. Further, ten open problems for the determination of $\mathbb{M}_{s}(x)$ were proposed.

In this paper, some results on $\mathbb{M}_{s}(x)$ are given first. Then we solve the following two open problems presented by Ding. Two classes of APN functions used to induce cyclic codes are

(1) The inverse APN function over finite field $G F(r)[10]: f(x)=x^{q^{m}-2}$;

(2) The Dobbertin APN function over finite field $G F\left(2^{5 i}\right)$ [11]: $f(x)=$ $x^{2^{4 i}+2^{3 i}+2^{2 i}+2^{i}-1}$.

The minimal polynomial of the inverse APN function with $p=2$ was solved in [9,20]. We generalize the result for a general odd prime $p$, which gives the corresponding cyclic code. In fact, this technique can be useful for other open problems presented by Ding.

\section{Preliminary}

In this section, some notations and results are introduced. Let $Z_{N}=\{0,1, \cdots$, $N-1\}$ with the integer addition modulo $N$ and integer multiplication modulo $N$ operation for a positive integer $N$. Let the $q$-cyclotomic coset containing $j$ modulo $N$ with the action $x \longmapsto q x \bmod N$ be defined by

$$
C_{j}=\left\{j, q j, q^{2} j, \ldots, q^{l_{j}-1}\right\} \subset Z_{N}
$$

where $l_{j}$ is the smallest positive integer such that $q^{l_{j}} j \equiv j \bmod N$ and $l_{j}$ is called the size of $C_{j}$. For a sequence over finite field $G F(q)$ with period $q^{m}-1$, we have the following result on the linear span and minimal polynomial [1].

Lemma 1 Let $s^{\infty}$ be a sequence with period $q^{m}-1$ over finite field $G F(q)$, which has a unique expansion of the form

$$
s_{t}=\sum_{i=0}^{q^{m}-2} c_{i} \alpha^{i t}, \forall t \geq 0,
$$


where $\alpha$ is a generator of $G F\left(q^{m}\right)^{*}$, and $c_{i} \in G F\left(q^{m}\right)$. Let $I=\left\{i: c_{i} \neq 0\right\}$, then the minimal polynomial of $s^{\infty}$ is

$$
\mathbb{M}_{s}(x)=\prod_{i \in I}\left(1-\alpha^{i} x\right)
$$

and the linear span $\mathbb{L}_{s}$ of $s^{\infty}$ is $|I|$.

From this lemma, in order to determine the minimal polynomial and linear span, we just need to compute the expansion of $s_{t}$. The Lucas's theorem [15. can be a useful tool in the computation.

Theorem 1 Let $N=n_{t} q^{t}+n_{t-1} q^{t-1}+\cdots+n_{1} q+n_{0}$ and $M=m_{t} q^{t}+$ $m_{t-1} q^{t-1}+\cdots+m_{1} q+m_{0}$, then

$$
\left(\begin{array}{c}
N \\
M
\end{array}\right) \equiv\left(\begin{array}{c}
n_{t} \\
m_{t}
\end{array}\right)\left(\begin{array}{c}
n_{t-1} \\
m_{t-1}
\end{array}\right) \cdots\left(\begin{array}{c}
n_{0} \\
m_{0}
\end{array}\right) \bmod p
$$

Two most important classes of nonlinear functions in cryptography are almost perfect nonlinear (APN) functions and perfect nonlinear (or planar) functions [2, 18, which can be used to construct cyclic codes [4,5,6,9]. A function $f$ : $G F(r) \longrightarrow G F(r)$ is called a planar function if

$$
\max _{a \in G F(r)^{*}, b \in G F(r)} \#(\{x \in G F(r): f(x+a)-f(x)=b\})=1 .
$$

And a function $f: G F(r) \longrightarrow G F(r)$ is called an almost perfect nonlinear $(\mathrm{APN})$ function if

$$
\max _{a \in G F(r)^{*}, b \in G F(r)} \#(\{x \in G F(r): f(x+a)-f(x)=b\})=2 .
$$

The APN functions considered in this paper are

- The inverse APN function [10]: $f(x)=x^{q^{m}-2}$;

- The Dobbertin APN function [11: $f(x)=x^{2^{4 i}+2^{3 i}+2^{2 i}+2^{i}-1}, m=5 i$.

\section{The minimal polynomial of sequence from binomial functions}

In this section, we analyze the determination of the minimal polynomial of the sequence $s^{\infty}$ with $s_{t}=\operatorname{Tr}\left(\left(1+\alpha^{t}\right)^{e}\right)$, where $0 \leq e \leq q^{m}-2$. Further, Let 
$n=q^{m}-1$ for the rest of the paper.

$$
\begin{aligned}
s_{t} & =\operatorname{Tr}\left(\left(1+\alpha^{t}\right)^{e}\right) \\
& =\operatorname{Tr}\left(\sum_{i=0}^{q^{m}-2}\left(\begin{array}{l}
e \\
i
\end{array}\right) \alpha^{i t}\right) \\
& =\sum_{i=0}^{q^{m}-2}\left(\begin{array}{l}
e \\
i
\end{array}\right) \sum_{j=0}^{m-1} \alpha^{q^{j} i t} \\
& =\sum_{i=0}^{q^{m}-2}\left(\sum_{j=0}^{m-1}\left(\begin{array}{ll}
q^{j} i & \bmod n
\end{array}\right) \bmod p\right) \alpha^{i t} \\
& =\sum_{i=0}^{q^{m}-2} C_{e, q, m}(i) \alpha^{i t}
\end{aligned}
$$

where $C_{e, q, m}(i)=\sum_{j=0}^{m-1}\left(\begin{array}{cc}q_{q^{j}} & e \\ \bmod n\end{array}\right) \bmod p$.

Define

$$
\operatorname{Supp}\left(C_{e, q, m}\right)=\left\{i: 0 \leq i<n, C_{e, q, m}(i) \neq 0\right\} .
$$

From the definition of $C_{e, q, m}(i)$, we have

$$
C_{e, q, m}\left(q^{j} i\right)=C_{e, q, m}(i) .
$$

Then there exists a subset $\widetilde{\operatorname{Supp}}\left(C_{e, q, m}\right)$ of $\operatorname{Supp}\left(C_{e, q, m}\right)$ satisfying

$$
\operatorname{Supp}\left(C_{e, q, m}\right)=\underset{i \in \widehat{\operatorname{Supp}}\left(C_{e, q, m}\right)}{\bigcup}\left\{q^{j} i \bmod n: j=0,1, \cdots, m-1\right\} .
$$

Further, we can choose $\widetilde{\operatorname{Supp}}\left(C_{e, q, m}\right)$ with the condition that if $i, i^{\prime} \in \widetilde{\operatorname{Supp}}\left(C_{e, q, m}\right)$ and $j=1, \cdots, m-1, n \nmid\left(i^{\prime}-q^{j} i\right)$. Then

$$
\begin{aligned}
s_{t} & =\operatorname{Tr}\left(\left(1+\alpha^{t}\right)^{e}\right) \\
& =\sum_{i \in \operatorname{Supp}\left(C_{e, q, m}\right)} C_{e, q, m}(i) \alpha^{i t} \\
& =\sum_{i \in \widehat{\operatorname{Supp}}\left(C_{e, q, m}\right)} \sum_{i^{\prime} \in C_{i}} C_{e, q, m}\left(i^{\prime}\right) \alpha^{i^{\prime} t}
\end{aligned}
$$

where $C_{i}$ is the $q$-cyclotomic coset containing $i$ modulo $n=q^{m}-1$, that is , $C_{i}=\left\{q^{j} i \bmod n: j=0,1, \cdots, m-1\right\}$.

From the above discussion, we have the following theorem.

Theorem 2 Let $s^{\infty}$ be a sequence over $G F(q)$ defined by $s_{t}=\operatorname{Tr}\left(\left(1+\alpha^{t}\right)^{e}\right)$. Then the linear span $\mathbb{L}_{s}$ of $s^{\infty}$ is \# $\left(\operatorname{Supp}\left(C_{e, q, m}\right)\right)$, and the minimal polynomial of $s^{\infty}$ is

$$
\mathbb{M}_{s}(x)=\prod_{\gamma \in \operatorname{Supp}\left(C_{e, q, m}\right)}\left(x-\gamma^{-1}\right)=\prod_{\gamma \in \widehat{\operatorname{Supp}}\left(C_{e, q, m}\right)} m_{\gamma^{-1}}(x),
$$

where $m_{\gamma^{-1}}(x)$ is the minimal polynomial of $\gamma^{-1}$ over $G F(q)$. 
Proof From Identity (10) and Lemma 1 this theorem follows.

4 Cyclic codes from APN function: $x^{q^{m}-2}$

Note that $\left(\begin{array}{c}N-1 \\ M\end{array}\right)=\frac{N-M}{N}\left(\begin{array}{l}N \\ M\end{array}\right)$, then

$$
\begin{aligned}
C_{e, q, m}(i) & \left.\equiv \sum_{i^{\prime} \equiv q^{j} i} \quad \begin{array}{c}
e \\
\bmod n, j \in Z_{m} \\
i^{\prime}
\end{array}\right) \\
& \equiv \sum_{i^{\prime} \equiv q^{j} i} \frac{e+1-i^{\prime}}{\bmod n, j \in Z_{m}}\left(\begin{array}{c}
e+1 \\
i^{\prime}
\end{array}\right) \\
& \equiv \sum_{i^{\prime} \equiv q^{j} i} \sum_{\bmod n, j \in Z_{m}}\left(1+i^{\prime}\right)\left(\begin{array}{c}
e+1 \\
i^{\prime}
\end{array}\right) \bmod p
\end{aligned}
$$

Let $i=\sum_{j=0}^{m-1} i_{j} q^{j}$, then $q i \bmod n=i_{m-1}+i_{0} q+\cdots+i_{m-2} q^{m-1}$. From $e+1=\sum_{j=0}^{m-1}(q-1) q^{j}$ and Lucas's theorem, we have

$$
\begin{aligned}
\left(\begin{array}{c}
e+1 \\
q i \\
\bmod n
\end{array}\right) & \equiv\left(\begin{array}{c}
q-1 \\
i_{m-2}
\end{array}\right) \cdots\left(\begin{array}{c}
q-1 \\
i_{0}
\end{array}\right)\left(\begin{array}{c}
q-1 \\
i_{m-1}
\end{array}\right) \\
& \equiv\left(\begin{array}{c}
q-1 \\
i_{m-1}
\end{array}\right) \cdots\left(\begin{array}{c}
q-1 \\
i_{1}
\end{array}\right)\left(\begin{array}{c}
q-1 \\
i_{0}
\end{array}\right) \\
& \equiv\left(\begin{array}{c}
e+1 \\
i
\end{array}\right) \bmod p
\end{aligned}
$$

For $i^{\prime}=q^{j} i \bmod n\left(j \in Z_{m}\right),\left(\begin{array}{c}e+1 \\ i^{\prime}\end{array}\right) \equiv\left(\begin{array}{c}e+1 \\ i\end{array}\right) \bmod p$. Then we get

$$
\begin{aligned}
C_{e, q, m}(i) & \equiv\left(\begin{array}{c}
e+1 \\
i
\end{array}\right) \sum_{i^{\prime} \equiv q^{j} i} \sum_{\bmod n, j \in Z_{m}}\left(1+i^{\prime}\right) \\
& \equiv\left(\begin{array}{c}
e+1 \\
i
\end{array}\right)\left(\sum_{j=0}^{m-1} i_{j}+m\right) \bmod p
\end{aligned}
$$

where $i_{j} \equiv q^{j} i \bmod n$. Let $s_{q}(i)=\sum_{j=0}^{m-1} i_{j}$, then

$$
\operatorname{Supp}\left(C_{e, q, m}\right)=\left\{i \in Z_{n}: s_{q}(i)+m \not \equiv 0 \bmod p\right\} .
$$

Further, we have

$$
\#\left(\operatorname{Supp}\left(C_{e, q, m}\right)\right)=q^{m}\left(1-\frac{1}{p}\right) .
$$


Theorem 3 Let $s^{\infty}$ be a sequence defined by $s_{t}=\operatorname{Tr}\left(\left(1+\alpha^{t}\right)^{q^{m}-2}\right)$. Then the linear span $\mathbb{L}_{s}$ of $s^{\infty}$ is $q^{m}\left(1-\frac{1}{p}\right)$, and the minimal polynomial of $s^{\infty}$ is

$$
\mathbb{M}_{s}(x)=\prod_{\substack{i \in Z_{n} \\ s_{q}(i)+m \neq j \\ m \neq 0}}\left(x-\alpha^{-i}\right),
$$

where $s_{q}(i)=\sum_{j=0}^{m-1} i_{j}$.

Proof From Identities (13), (14) and Theorem 2, this theorem follows.

Theorem 4 The cyclic code $\mathcal{C}_{s}$, induced by the sequence in Theorem 3 is an $\left[n, \frac{q^{m}}{p}-1, d\right]$ code, whose generator polynomial is given by Identity (15). Further, $d \geq \max \left\{2 p-1, \frac{q(p-1)}{p}+1\right\}$.

Proof The dimension of $\mathcal{C}_{s}$ can be obtained by Theorem 3 . Then we consider the lower bound of minimum weight $d$. Note that the weight distribution of the cyclic code generated by $\mathbb{M}_{s}(x)$ is the same as that of the cyclic code generated by the reciprocal polynomial of $\mathbb{M}_{s}(x)$. The reciprocal polynomial of $\mathbb{M}_{s}(x)$ has zero points $\alpha^{j}$, where $j \in\{q-p+1, q-p+2, \cdots, q+p-2\}$. From the BCH bound, we have $d \geq 2 p-1$. Further, the reciprocal polynomial of $\mathbb{M}_{s}(x)$ has zero points $\alpha^{p j+1}$, where $j \in\left\{0,1, \cdots, \frac{q(p-1)}{p}-1\right\}$. From the Hartmann-Tzeng bound [13, we have $d \geq \frac{q(p-1)}{p}+1$. Hence, $d \geq \max \left\{2 p-1, \frac{q(p-1)}{p}+1\right\}$.

Example 1 Let $q=3$ and $m=2$. Let $\alpha$ be a primitive element of $G F\left(3^{2}\right)$ satisfying $\alpha^{2}+2 \alpha+2=0$. The the corresponding cyclic code $\mathcal{C}_{s}$ is an $[8,2,6]$ code, whose generator polynomial is

$$
\mathbb{M}_{s}(x)=x^{6}+2 x^{5}+2 x^{4}+2 x^{2}+x+1 \text {. }
$$

And its dual is an $[8,6,2]$ cyclic code.

Example 2 Let $q=3$ and $m=3$. Let $\alpha$ be a primitive element of satisfying $\alpha^{3}+2 \alpha+1=0$. The corresponding cyclic code $\mathcal{C}_{s}$ is an $[26,8,10]$ code, whose generator polynomial is

$$
\begin{aligned}
\mathbb{M}_{s}(x)= & x^{18}+2 x^{16}+2 x^{15}+x^{14}+x^{12}+x^{11}+x^{10}+ \\
& x^{9}+x^{8}+x^{7}+x^{6}+x^{4}+2 x^{3}+2 x^{2}+1 .
\end{aligned}
$$

And its dual is an $[26,18,4]$ cyclic code.

5 Cyclic codes from the APN function: $x^{2^{4 i}+2^{3 i}+2^{2 i}+2^{i}-1}$

Let $m=5 i$ and $e=2^{4 i}+2^{3 i}+2^{2 i}+2^{i}-1$. The binary sequence of $e$ is

$$
\underline{e}=\underbrace{00 \cdots 1}_{i} \underbrace{00 \cdots 1}_{i} \underbrace{00 \cdots 1}_{i} \underbrace{00 \cdots 0}_{i} \underbrace{11 \cdots 1}_{i},
$$


which has $m$ bits.

For an integer $x \in\{0,1, \cdots, n-1\}$, the binary expansion of $x$ is $\sum_{j=0}^{m-1} x_{j} 2^{j}$, and the corresponding binary sequence is $\underline{x}=x_{m-1} x_{m-1} \cdots x_{0}$ with $m$ bits. Then $\underline{x}_{i}$ and $x_{i}$ are the same. Let $S t$ be the set of all the binary sequences with $m$ length except $1^{m}=\underbrace{11 \cdots 1}_{m}$. Let $G$ be the group with elements of cyclically shift transformations on $\stackrel{m}{S t}$. Then $G$ is a cyclic code with order $m$. Let $\tau \in G$ and $\tau\left(b_{m-1} b_{m-2} \cdots b_{0}\right)=b_{0} b_{m-1} \cdots b_{1}$, then $G=\langle\tau\rangle$. Note that there is a bijection map between $Z_{n}$ and $S t$. Then $\tau$ is corresponding to an action $x \longmapsto q^{m-1} x$ over $Z_{n}$, that is, a $q-$ cyclotomic action on $Z_{n}$ is corresponding to a cyclic action on $S t$.

Definition 1 Let $\underline{x}, \underline{y} \in S t$. It is said that $\underline{x}$ is covered by $\underline{y}$ or $\underline{y}$ is a cover of $\underline{x}$, if for $\underline{x}_{i}=1$, then $\underline{y}_{i}=1$. It is denoted by that $\underline{x} \preceq \underline{y}$.

Definition 2 Let $\underline{x} \in S t$. It is said that $\underline{x}$ is cyclically covered by $\underline{e}$, if there exists an element $\sigma \in G$ satisfying $\sigma(\underline{x}) \preceq \underline{e}$. If such $\sigma$ does not exist, then $\underline{x}$ is not cyclically covered by $\underline{\underline{e}}$.

Definition 3 Let $\underline{x} \in S t$. the sequence $\underline{x}$ is called an even sequence if $\#\{\sigma \in$ $G \mid \sigma(\underline{x}) \preceq \underline{e}\}$ is even. Otherwise, $\underline{x}$ is an odd sequence.

Lemma 2 Let $m=5 i$ and $e=2^{4 i}+2^{3 i}+2^{2 i}+2^{i}-1$. Let $p=q=2, r=2^{m}$ and $x \in Z_{n}$. Then $x \in \operatorname{Supp}\left(C_{e, q, m}\right)$ if and only if $\underline{x}$ is an odd sequence.

Proof From Lucas's theorem, we have

$$
\begin{aligned}
C_{e, q, m}(x) & =\sum_{x^{\prime}=q^{j} x, j \in Z_{m}}\left(\begin{array}{c}
e \\
x^{\prime}
\end{array}\right) \\
& =\sum_{\underline{x}^{\prime}=\sigma(\underline{x}), \sigma \in G} \prod_{k=0}^{m-1}\left(\begin{array}{c}
\underline{e}_{k} \\
\underline{x}_{k}^{\prime}
\end{array}\right) \\
& =\#(\{\sigma \in G: \sigma(\underline{x}) \preceq \underline{e}\}) \bmod 2 .
\end{aligned}
$$

Hence, $C_{e, q, m} \neq 0$ if and only if $\underline{x}$ is an odd sequence.

Lemma 3 Let $l \geq 2$ and $\underline{y}=\underbrace{00 \cdots 0}_{i} \underbrace{00 \cdots 0}_{i} \underbrace{00 \cdots 0}_{i} \underbrace{00 \cdots 0}_{i} \underbrace{1 * \cdots * 1}_{l} \underbrace{0 \cdots 0}_{i-l}$, where $*$ is any element in $\{0,1\}$. Then $\#\{\sigma(\underline{y}) \mid \sigma \in G\}=m$.

Proof We just need to prove that if $\sigma \neq i d$, then $\sigma(\underline{y}) \neq \underline{y}$. Let $\sigma=\tau^{j}$, where $0<j<m$.

(1) If $0<j \leq i-l$, then

$$
(\sigma(\underline{y}))_{i-l-j}=\underline{y}_{i-l}=1 .
$$

Further, $0 \leq i-l-j<i-l$. Then

$$
\underline{y}_{i-l-j}=0 \text {. }
$$


Hence,

$$
(\sigma(\underline{y}))_{i-l-j} \neq \underline{y}_{i-l-j} .
$$

(2) If $i-l<j \leq m-l$, then

$$
(\sigma(\underline{y}))_{m+i-l-j}=\underline{y}_{i-l}=1 .
$$

Further, $i \leq m+i-l-j \leq m-1$. Then

$$
\underline{y}_{m+i-l-j}=0 \text {. }
$$

Hence,

$$
(\sigma(\underline{y}))_{m+i-l-j} \neq \underline{y}_{m+i-l-j} .
$$

(3) If $m-l<j<m$, then

$$
(\sigma(\underline{y}))_{m+i-1-j}=\underline{y}_{i-1}=1 .
$$

Further, $i-1<m+i-1-j<i+l-1$. Then

$$
\underline{y}_{m+i-1-j}=0 \text {. }
$$

Hence,

$$
(\sigma(\underline{y}))_{m+i-1-j} \neq \underline{y}_{m+i-1-j} .
$$

Consequently, when $0<j<m, \sigma(\underline{y})=\tau^{j}(\underline{y}) \neq \underline{y}$. This lemma follows.

If $l \geq 2$, denote the set of all the elements like $\underline{y}$ in Lemma 3 by $\mathcal{R}_{l}^{1}$, that is,

$$
\mathcal{R}_{l}^{1}=\{\underline{y} \in S t: \underline{y}=\underbrace{00 \cdots 0}_{4 i} \underbrace{1 * \cdots * 1}_{l} \underbrace{00 \cdots 0}_{i-l}\} \text {. }
$$

Lemma 4 Let $y \in \mathcal{R}_{l}^{1}$. Let $\sigma=\tau^{j}$, where $0 \leq j \leq i-1$. Then $\sigma(y) \preceq \underline{e}$ if and only if $0 \leq \bar{j} \leq i-l$. Further, $y$ is an odd sequence if and only if $i-l+1$ is odd.

Proof From $0 \leq j \leq i-l, \sigma(\underline{y}) \preceq \underline{e}$. Further, we have

From $\sigma(\underline{y}) \preceq \underline{e}$

$$
\begin{gathered}
(\sigma(\underline{y}))_{i-1-j}=\underline{y}_{i-1}=1, \\
(\sigma(\underline{y}))_{i-l-j}=\underline{y}_{i-l}=1 .
\end{gathered}
$$

$$
\underline{e}_{i-1-j}=\underline{e}_{i-l-j}=1 .
$$

Hence, the $(i-1-j)$-th element of $y$ is 1 . Further, from the $(i-1-j)$-th element, the $(l-1)$-th element is also 1 . Then

$$
i-1-j(\bmod m) \in\{i-1, i-2, \cdots, l-1\},
$$

Hence,

$$
j \in\{0,1, \cdots, i-l\} .
$$

This lemma follows. 
Lemma 5 The set of all the odd sequences in $\bigcup_{2 \leq l \leq i} \mathcal{R}_{l}^{1}$ is

$$
\mathcal{R}^{1}=\bigcup_{2 \leq l \leq i, l \equiv i} \mathcal{R}_{l}^{1}
$$

Further,

$$
\#\left(\mathcal{R}^{1}\right)= \begin{cases}\frac{2^{i}-1}{3}, & i \text { is even }, \\ \frac{2^{i}-2}{3}, & i \text { is odd. }\end{cases}
$$

Proof From Lemma 4, the set of all the odd sequence in $\bigcup_{2 \leq l \leq i} \mathcal{R}_{l}^{1}$ is

$$
\mathcal{R}^{1}=\bigcup_{2 \leq l \leq i, l \equiv i} \mathcal{R}_{l}^{1}
$$

Further, $\#\left(\mathcal{R}_{l}^{1}\right)=2^{l-2}$. When $l \neq l^{\prime}, \mathcal{R}_{l}^{1} \cap \mathcal{R}_{l^{\prime}}^{1}=\emptyset$.

If $i$ is even,

$$
\#\left(\mathcal{R}^{1}\right)=2^{0}+2^{2}+2^{4}+\cdots+2^{i-2}=\frac{2^{i}-1}{3} .
$$

If $i$ is odd,

$$
\#\left(\mathcal{R}^{1}\right)=2^{1}+2^{3}+2^{5}+\cdots+2^{i-2}=\frac{2^{i}-2}{3} .
$$

This lemma follows.

Lemma 6 Let $\mathcal{R}^{2}$ be the set of all the elements y in St satisfying the following conditions:

(1) If $j \geq i$ and $j \notin\{2 i, 3 i, 4 i\}, \underline{y}_{j}=0$;

(2) There exists $j \in\{2 i, 3 i, 4 i\}$ satisfying $\underline{y}_{j}=1$;

(3) \#(\{j:0 $\left.\left.\leq j \leq i-1, \underline{y}_{j}=1\right\}\right) \geq 2$.

For any $\underline{y} \in \mathcal{R}^{2}$, let $\sigma \in G$ satisfying $\sigma(\underline{y}) \preceq \underline{e}$. Then $\sigma=1$.

Proof From Condition (3), choose $j_{1}$ and $j_{2}$ satisfying

$$
0 \leq j_{1}<j_{2} \leq i-1, \underline{y}_{j_{1}}=\underline{y}_{j_{2}}=1 .
$$

Let $\sigma=\tau^{j}$ and $\sigma(\underline{y}) \preceq \underline{e}$, then

$$
\begin{gathered}
\tau^{j}(\underline{y})_{j_{1}-j}=\underline{y}_{j_{1}}=1=\underline{e}_{j_{1}-j}, \\
\tau^{j}(\underline{y})_{j_{2}-j}=\underline{y}_{j_{2}}=1=\underline{e}_{j_{2}-j} .
\end{gathered}
$$

Further

$$
1 \leq\left(j_{2}-j\right)-\left(j_{1}-j\right) \leq i-1
$$

From the sequence $\underline{e}$

$$
j_{2}-j(\bmod m) \in\{1,2, \cdots, i-2, i-1\},
$$


that is,

$$
j=j_{2}-(i-1), j_{2}-(i-2), \cdots, j_{2}-1 \text {. }
$$

Further,

$$
-(i-2) \leq j \leq i-2
$$

From Condition (2), just choose $j_{0} \in\{2 i, 3 i, 4 i\}$ sastisfying $\underline{y}_{j_{0}}=1$. Note that

$$
\tau^{j}\left(\underline{y}_{j_{0}-j}=\underline{y}_{j_{0}}=1\right. \text {. }
$$

Hence,

$$
\underline{e}_{j}-j=1 \text {. }
$$

We can have $j=0$, that is, $\sigma=1$.

Lemma 7 Let $\mathcal{R}^{2}$ be defined above. Then $\#\left(\mathcal{R}^{2}\right)=7\left(2^{i}-i-1\right)$.

Proof This lemma can be obtained by the definition of $\mathcal{R}^{2}$.

Lemma 8 Let $\mathcal{R}_{4}=\left\{y \in S t: y \preceq \underline{e}, w t(y)=4, y \notin \mathcal{R}^{1} \cup \mathcal{R}^{2}\right\}$. Then $\#\left(\mathcal{R}_{4}\right)=i$. Further, let $\sigma \in G$ satisfying $\sigma(\underline{y}) \preceq \underline{e}$ for any $\underline{y} \in \mathcal{R}_{4}$, then $\sigma=1$.

Proof From the definition of $\mathcal{R}_{4}, \underline{y} \in \mathcal{R}_{4}$ if and only if

$$
\left\{j: i \leq j \leq 5 i-1, \underline{y}_{j}=1\right\}=\{2 i, 3 i, 4 i\}, \#\left(\left\{j: 0 \leq j \leq i-1, \underline{y}_{j}=1\right\}\right)=1 .
$$

Hence,

$$
\#\left(\mathcal{R}_{4}\right)=i
$$

Let $\underline{y} \in \mathcal{R}_{4}$ and $\sigma(\underline{y}) \preceq \underline{e}$. Then

$$
\sigma(\underline{y}) \in \mathcal{R}_{4}
$$

Let $\sigma=\tau^{j}$. Then

$\#(\{(2 i-j) \bmod 5 i,(3 i-j) \bmod 5 i,(4 i-j) \bmod 5 i\} \bigcap\{2 i, 3 i, 4 i\}) \geq 2$

Hence,

$$
j \in\{0, i,-i, 2 i,-2 i\} \text {. }
$$

When $j= \pm i, \pm 2 i, \tau^{j}(\underline{y}) \npreceq \underline{e}$. Hence, $j=0$, that is, $\sigma=1$.

Lemma 9 Let $\mathcal{R}_{3}=\left\{\underline{y} \in S t: \underline{y} \preceq \underline{e}, w t(\underline{y})=3, \underline{y}\right.$ is odd, $\left.\underline{y} \notin \mathcal{R}^{1} \cup \mathcal{R}^{2}\right\}$. Then $\#\left(\mathcal{R}_{3}\right)=3(i-1)$. Further, let $\sigma \in G$ satisfying $\sigma(\underline{y}) \preceq \underline{\text { e for }}$ any $\underline{y} \in \mathcal{R}_{3}$. Then $\sigma=1$. 
Proof From the definition of $\mathcal{R}_{3}$, an element $\underline{y}$ in $\mathcal{R}_{3}$ satisfies one of the following three conditions:

(1) $\underline{y}_{2 i}=\underline{y}_{3 i}=\underline{y}_{4 i}=1$;

(2) $\#\left(\left\{j: \underline{y}_{j}=\overline{1}_{1}, j \in\{2 i, 3 i, 4 i\}\right\}\right)=2, \underline{y}_{0}=1$;

(3)\# $\left(\left\{j: \underline{y}_{j}=1, j \in\{2 i, 3 i, 4 i\}\right\}\right)=2, \#\left(\left\{j: \underline{y}_{j}=1,1 \leq j \leq i-1\right\}\right)=1$.

We will prove that $y$ satisfying one of Condition (1) and (2) is even, and $\underline{y}$ in Condition (3) is odd.

$y$ in Condition (1) or (2) can fall into the following four cases:

(a) $\underline{y}_{2 i}=\underline{y}_{3 i}=\underline{y}_{4 i}=1$. It is denoted by $\underline{y}^{(1)}$;

(b) $\underline{y}_{3 i}=\underline{y}_{4 i}=\underline{y}_{0}=1$. It is denoted by $\underline{y}^{(2)}$;

(c) $\underline{y}_{2 i}=\underline{y}_{3 i}=\underline{y}_{0}=1$. It is denoted by $\underline{y}^{(3)}$;

(d) $\underline{y}_{4 i}=\underline{y}_{2 i}=\underline{y}_{0}=1$. It is denoted by $\underline{y}^{(4)}$.

$\underline{y}^{(1)}$ satisfies that $\left\{\sigma \in G: \sigma\left(\underline{y}^{(1)}\right) \preceq \underline{e}\right\}=\left\{\tau^{0}, \tau^{4 i}\right\}$. Hence, $\underline{y}^{(1)}$ is even. Since $\tau^{4 i}\left(\underline{y}^{(1)}\right)=\underline{y}^{(2)}, \underline{y}^{(2)}$ is also even.

$\underline{y}^{(3)}$ satisfies $\left\{\sigma \in \bar{G}: \sigma\left(\underline{y}^{(3)}\right) \preceq \underline{e}\right\}=\left\{\tau^{0}, \tau^{3 i}\right\}$. Hence, $\underline{y}^{(3)}$ is even. Since $\tau^{3 i}\left(\underline{y}^{(3)}\right)=\underline{y}^{(4)}, \underline{y}^{(4)}$ is also even.

Consequently, $\underline{y}$ in Condition (1) or (2) is an even sequence.

$\underline{y}$ in Condition (3) satisfies

$$
\{\sigma \in G: \sigma(\underline{y}) \preceq \underline{e}\}=\left\{\tau^{0}\right\} .
$$

Hence, $y$ in Condition (3) is odd. Further, all the sequences in $\mathcal{R}_{3}$ fall into sequences in Condition (3). Then, $\#\left(\mathcal{R}_{3}\right)=3(i-1)$.

The proof of $\sigma=1$ is similar to that in Lemma 8 .

Lemma 10 Let $\mathcal{R}_{2}=\left\{\underline{y}^{(1)}, \underline{y}^{(2)}\right\}$, where

$$
\begin{aligned}
& \underline{y}^{(1)}=\underbrace{00 \cdots 01}_{i} \underbrace{00 \cdots 01}_{i} \underbrace{00 \cdots 00}_{i} \underbrace{00 \cdots 00}_{i} \underbrace{00 \cdots 00}_{i}, \\
& \underline{y}^{(2)}=\underbrace{00 \cdots 01}_{i} \underbrace{00 \cdots 00}_{i} \underbrace{00 \cdots 01}_{i} \underbrace{00 \cdots 00}_{i} \underbrace{00 \cdots 00}_{i} .
\end{aligned}
$$

Let $\mathcal{R}_{2}^{\prime}=\left\{\underline{y} \in S t: \underline{y} \preceq \underline{e}, w t(\underline{y})=2, \underline{y}\right.$ is odd, $\left.\underline{y} \notin \mathcal{R}^{1} \cup \mathcal{R}^{2}\right\}$. Then $\underline{y}^{(1)}$ is not cyclically equivalent to $\underline{y}^{(2)}$. Further, $\overline{\mathcal{R}}_{2}^{\prime}=\left\{\underline{y}^{(\overline{1})}, \tau^{i}\left(\underline{y}^{(1)}\right), \tau^{4 i}\left(\underline{y}^{(1)}\right), \underline{y}^{(\overline{2)}}, \tau^{2 i}\left(\underline{y}^{(2)}\right), \tau^{4 i}\left(\underline{y}^{(2)}\right)\right\}$.

Proof It is obvious that $\underline{y}^{(1)}$ and $\underline{y}^{(2)}$ are not cyclically equivalent. Note that

$$
\begin{gathered}
\left\{\sigma \in G: \sigma\left(\underline{y}^{(1)}\right) \preceq \underline{e}\right\}=\left\{\tau^{0}, \tau^{i}, \tau^{4 i}\right\}, \\
\left\{\sigma \in G: \sigma\left(\underline{y}^{(2)}\right) \preceq \underline{e}\right\}=\left\{\tau^{0}, \tau^{2 i}, \tau^{4 i}\right\} .
\end{gathered}
$$

Then $\underline{y}^{(1)}, \underline{y}^{(2)} \in \mathcal{R}_{2}^{\prime}$. From the definition of $\mathcal{R}_{2}^{\prime}, \tau^{i}\left(\underline{y}^{(1)}\right) 4, \tau^{4 i}\left(\underline{y}^{(1)}\right), \tau^{2 i}\left(\underline{y}^{(2)}\right)$, $\tau^{4 i}\left(\underline{y}^{(\overline{2})}\right) \in \mathcal{R}_{2}^{\prime}$. To complete the proof, we just show that $\underline{y}$ in the following three conditions does not lie in $\mathcal{R}_{2}^{\prime}$.

(1) $w t(\underline{y})=2, \underline{y}_{4 j}=\underline{y}_{j}=1,0<j \leq i-1$. It is denoted by $\underline{y}^{(3)}$; 
(2) $w t(\underline{y})=2, \underline{y}_{2 j}=\underline{y}_{j}=1,0<j \leq i-1$. It is denoted by $\underline{y}^{(4)}$;

(3) $w t(\underline{y})=2, \underline{y}_{3 j}=\underline{y}_{j}=1,0<j \leq i-1$. It is denoted by $\underline{y}^{(5)}$.

Note that

$$
\begin{aligned}
& \left\{\sigma \in G: \sigma\left(\underline{y}^{(3)}\right) \preceq \underline{e}\right\}=\left\{\tau^{0}, \tau^{3 i+j}\right\}, \\
& \left\{\sigma \in G: \sigma\left(\underline{y}^{(4)}\right) \preceq \underline{e}\right\}=\left\{\tau^{0}, \tau^{i+j}\right\}, \\
& \left\{\sigma \in G: \sigma\left(\underline{y}^{(5)}\right) \preceq \underline{e}\right\}=\left\{\tau^{0}, \tau^{2 i+j}\right\},
\end{aligned}
$$

Hence, $\underline{y}^{(3)}, \underline{y}^{(4)}$ and $\underline{y}^{(5)}$ are even. Consequently, this lemma follows.

If $i$ is an odd integer, any sequence with weight 1 is an even sequence. If $i$ is even, any sequence with weight 1 is an odd sequence. However, the zero sequence is odd if $i$ is odd and it is even if $i$ is even. For convenience, some notations are given below.

$$
\begin{gathered}
\mathcal{R}_{1}= \begin{cases}\{\underbrace{00 \cdots 0}_{m-1} 1\}, & i \text { is even, } \\
\emptyset, & i \text { is odd. }\end{cases} \\
\mathcal{R}_{0}= \begin{cases}\emptyset, & i \text { is even, } \\
\{\underbrace{00 \cdots 0}_{m}\}, & i \text { is odd. }\end{cases} \\
\mathcal{R}=\mathcal{R}^{1} \bigcup \mathcal{R}^{2} \bigcup \mathcal{R}_{4} \bigcup \mathcal{R}_{3} \bigcup \mathcal{R}_{2} \bigcup \mathcal{R}_{1} \bigcup \mathcal{R}_{0} .
\end{gathered}
$$

Then we have the following lemma.

Lemma 11 Let $\mathcal{R}$ be defined above. Then

(1) $\#(\mathcal{R})=\left\{\begin{array}{ll}\frac{22}{3}\left(2^{i}-1\right)-3 i, & i \text { is even } \\ \frac{22}{3}\left(2^{i}-2\right)-3 i+7, & i \text { is odd }\end{array} ;\right.$

(2) Let $\sigma \in G$ satisfy $\sigma(\underline{x}) \in \mathcal{R}$ for some $\underline{x} \in \mathcal{R}$. Then $\sigma=\tau^{0}$;

(3) Let $\underline{x} \in \mathcal{R}$ and $x \neq 0^{m}$. Then $\#(\{\sigma(\underline{x}): \sigma \in G\})=m$;

(4) If $x \in \operatorname{Supp}\left(C_{e, q, m}\right)$, there exists $\sigma \in G$ satisfying $\sigma(\underline{x}) \in \mathcal{R}$.

Further,

$$
\widetilde{\operatorname{Supp}}\left(C_{e, q, m}\right)=\left\{x \in Z_{n}: \underline{x} \in \mathcal{R}\right\},
$$

and

$$
\#\left(\operatorname{Supp}\left(C_{e, q, m}\right)\right)= \begin{cases}m\left[\frac{22}{3}\left(2^{i}-1\right)-3 i\right], & i \text { is even } \\ m\left[\frac{22}{3}\left(2^{i}-2\right)-3 i+6\right]+1, & i \text { is odd }\end{cases}
$$


Proof Result (1) can be obtained by Lemma 5, Lemma 7, Lemma 8, Lemma 9 and definitions of $\mathcal{R}_{2}, \mathcal{R}_{1}, \mathcal{R}_{0}$.

Result (2) can be obtained by definitions of $\mathcal{R}^{1}, \mathcal{R}^{2}, \mathcal{R}_{4}, \mathcal{R}_{3}, \mathcal{R}_{2}, \mathcal{R}_{1}$ and $\mathcal{R}_{0}$.

Result (3) can be obtained by Lemma 5. Lemma 6, Lemma 8, Lemma 9 and definitions of $\mathcal{R}_{2}, \mathcal{R}_{1}, \mathcal{R}_{0}$.

Result (4) can be obtained by the definition of $\mathcal{R}$.

Finally, the result on $\widetilde{\operatorname{Supp}}\left(C_{e, q, m}\right)$ can be obtained by Result (2) and (4). The result on \# $\left.\operatorname{Supp}\left(C_{e, q, m}\right)\right)$ can be obtained by Result (1) and (3).

Theorem 5 Let $s^{\infty}$ be a sequence defined by $s_{t}=\operatorname{Tr}\left(\left(1+\alpha^{t}\right)^{2^{4 i}+2^{3 i}+2^{2 i}+2^{i}-1}\right)$. Then the linear span $\mathbb{L}_{s}$ of $s^{\infty}$ is

$$
\mathbb{L}_{s}= \begin{cases}m\left[\frac{22}{3}\left(2^{i}-1\right)-3 i\right], & i \text { is even }, \\ m\left[\frac{22}{3}\left(2^{i}-2\right)-3 i+6\right]+1, & i \text { is odd } .\end{cases}
$$

And the minimal polynomial of $s^{\infty}$ is

$$
\mathbb{M}_{s}(x)= \begin{cases}\prod_{i \in \mathcal{R}} m_{\alpha^{-i}}(x), & i \text { is even }, \\ (x-1) \prod_{i \in \mathcal{R}, i \neq 0^{m}} m_{\alpha^{-i}}(x), & i \text { is odd } .\end{cases}
$$

where $m_{\alpha^{-i}}(x)$ is the minimal polynomial of $\alpha^{-i}$.

Proof This theorem follows from Lemma 11 and Theorem 2.

Theorem 6 The binary cyclic code $\mathcal{C}_{s}$ induced by the sequence $s^{\infty}$ in Theorem 5 is an $\left[2^{m}-1,2^{m}-1-\mathbb{L}_{s}, d\right]$ code, whose generator polynomial is $\mathbb{M}_{s}(x)$ in Identity (22). $\mathbb{L}_{s}$ is given by Identity (21). Further,

$$
d \geq\left\{\begin{array}{ll}
2^{i}+1, i \equiv 0 & \bmod 2 \\
2^{i}+2, i \equiv 1 & \bmod 2
\end{array} .\right.
$$

Proof The dimension of $\mathcal{C}_{s}$ can be obtained by Theorem 5 and the definition of $\mathcal{C}_{s}$. Then we will give the lower bound of minimum weight.

Note that the weight distribution of the cyclic code generated by $\mathbb{M}_{s}(x)$ is the same as that of the cyclic code generated by the reciprocal polynomial of $\mathbb{M}_{s}(x)$. From Lemma 11, the reciprocal polynomial of $\mathbb{M}_{s}(x)$ has zero points $\alpha^{2^{4 i}+2^{3 i}+2^{2 i}+j}$, where $j \in\left\{0,1,2, \ldots 2^{i}-1\right\}$. From the BCH bound, we have $d \geq 2^{i}+1$. If $m$ is odd, then $\mathcal{C}_{s}$ is an even-weight code and $d \geq 2^{i}+2$.

Example 3 Let $q=2$ and $m=5$. Let $\alpha$ be a primitive element of $G F\left(2^{5}\right)$ satisfying $\alpha^{5}+\alpha^{2}+1=0$. Then the corresponding cyclic code $\mathcal{C}_{s}$ is an $[31,15,8]$ code, whose generator polynomial is

$$
\mathbb{M}_{s}(x)=x^{16}+x^{14}+x^{13}+x^{10}+x^{9}+x^{8}+x^{7}+x^{6}+x^{5}+x^{2}+x+1 .
$$

And its dual is an $[31,16,7]$ cyclic code.

Example 4 Let $q=2$ and $m=10$. Let $\alpha$ be a primitive element of $G F\left(2^{10}\right)$ satisfying $\alpha^{10}+\alpha^{6}+\alpha^{5}+\alpha^{3}+\alpha^{2}+\alpha+1=0$. Then the corresponding cyclic code $\mathcal{C}_{s}$ is an $[1023,863]$ code. And its dual is an $[1023,160]$ cyclic code. 


\section{Conclusion}

In this paper, we solve two open problems presented by Ding, which are on cyclic codes induced by the inverse APN functions and the Dobbertin APN functions. Further, we determine the dimensions and generator polynomials of these cyclic codes, which is from the determination of linear spans and minimal polynomials of sequences. The techniques we used in this paper are general and can be utilized in other open problems presented by Ding in 9 . Actually, we present a framework for determining the minimal polynomial of the sequence $s^{\infty}$ defined by $s_{t}=\operatorname{Tr}\left(\left(1+\alpha^{t}\right)^{e}\right)$.

Acknowledgements This work was supported by the National Natural Science Foundation of China (Grant Nos. 61272499, 10990011). Yanfeng Qi acknowledges support from Aisino Corporation Inc.

\section{References}

1. M. Antweiler, L. Bomer, "complex sequences over $G F\left(p^{M}\right)$ with a two-level autocorrelation function and a large linear span,"IEEE Trans. Inform. Theory, vol. 38, pp. 120-130, 1992.

2. T. Beth, C. Ding, "On almost perfect nonlinear permutations," in:Advances in Cryptology-EUROCRYPT 93,Lecture Notes in Comput. Sci. 765, Springer-Verlag, New York, 1993, pp. 6576

3. I. Blake, R. C. Mullen, The Mathematical Theory of Coding,Academic Press, New York, 1975.

4. A. Canteaut, P. Charpin, H. Dobbertin, "Weight divisibility of cyclic codes, highly nonlinear functions on $F_{2}$, and crosscorrelation of maximum-length sequences,"SIAM. J. Discrete Math., vol. 13, no. 1, pp. 105-138, 2000.

5. C. Carlet, P. Charpin, V. Zinoviev, "Codes, bent functions and permutations suitable for DES-like cryptosys-tems," Des. Codes Cryptogr.,vol. 15, pp. 125-156, 1998.

6. C. Carlet, C. Ding, J. Yuan, "Linear codes from highly nonlinear functions and their secret sharing schemes," IEEE Trans. Inform. Theory,vol. 51, no. 6, pp. 2089-2102, 2005.

7. P. Charpin, Open problems on cyclic codes, in: Handbook of Coding Theory, Part 1: Algebraic Coding, V.S. Pless, W. C. Huffman, and R. A. Brualdi, Eds. Amsterdam, The Netherlands: Elsevier, 1998, ch. 11.

8. R. T. Chien, "Cyclic decoding procedure for the Bose-Chaudhuri-Hocquenghem codes,"IEEE Trans. In-form. Theory, vol. 10, pp. 357-363, 1964.

9. C. Ding, "Cyclic codes from APN and planar functions," CoRR abs/1206.4687 ,2012.

10. T. Helleseth, C. Rong, D. Sandberg, "New families of almost perfect nonlinear power mappings,"IEEE Trans. Inform. Theory,vol. 45, no. 2, pp. 475-485, 1999.

11. H. Dobbertin, "Almost perfect nonlinear power functions on $G F\left(2^{n}\right)$ : The Niho case,"Inform. and Comput., vol. 151, pp. 57-72, 1999.

12. G. D. Forney, "On decoding BCH codes,"IEEE Trans. Inform. Theory, vol. 11, no. 4, pp. 549-557, 1995.

13. C. R. P. Hartmann, K. K. Tzeng, "Generalizations of the BCH bound,"Information and Control, vol. 20,pp. 489-498, 1972.

14. W. C. Huffman, V. Pless, Fundamentals of Error-Correcting Codes, Cambridge University Press, Cam-bridge, 2003.

15. E. Lucas, "Théorie des fonctions numériques simplement périodiques," Am. J. Math. 1, $229-231,1878$

16. L. Lidl, H. Niederreiter, Finite Fields, Cambridge University Press, Cambridge, 1997.

17. J. H. van Lint, R. M. Wilson, "On the minimum distance of cyclic codes,"IEEE Trans. Inform. Theory, vol. 32, no. 1, pp. 23-40, 1986. 
18. K. Nyberg, "Differentially uniform mappings for cryptography," in:Advances in Cryptology-EUROCRYPT 93,Lecture Notes in Comput. Sci. 765, Springer-Verlag, New York, 1993, pp. 55-64.

19. E. Prange, "Some cyclic error-correcting codes with simple decoding algorithms," Air Force Cambridge Research Center-TN-58-156, Cambridge, Mass., April 1958.

20. W. Si, C. Ding, "A simple stream cipher with proven properties,"Cryptography and Communications, to appear. 